\title{
Stabbing Simplices by Points and Flats
}

\author{
Boris Bukh · Jiří Matoušek • Gabriel Nivasch
}

Received: 28 April 2008 / Revised: 17 September 2008 / Accepted: 20 November 2008 /

Published online: 11 December 2008

(C) Springer Science+Business Media, LLC 2008

\begin{abstract}
The following result was proved by Bárány in 1982: For every $d \geq 1$, there exists $c_{d}>0$ such that for every $n$-point set $S$ in $\mathbb{R}^{d}$, there is a point $p \in \mathbb{R}^{d}$ contained in at least $c_{d} n^{d+1}-O\left(n^{d}\right)$ of the $d$-dimensional simplices spanned by $S$.

We investigate the largest possible value of $c_{d}$. It was known that $c_{d} \leq 1$ / $\left(2^{d}(d+1)\right.$ !) (this estimate actually holds for every point set $\left.S\right)$. We construct sets showing that $c_{d} \leq(d+1)^{-(d+1)}$, and we conjecture that this estimate is tight. The best known lower bound, due to Wagner, is $c_{d} \geq \gamma_{d}:=\left(d^{2}+1\right) /\left((d+1) !(d+1)^{d+1}\right)$; in his method, $p$ can be chosen as any centerpoint of $S$. We construct $n$-point sets with a centerpoint that is contained in no more than $\gamma_{d} n^{d+1}+O\left(n^{d}\right)$ simplices spanned by $S$, thus showing that the approach using an arbitrary centerpoint cannot be further improved.

We also prove that for every $n$-point set $S \subset \mathbb{R}^{d}$, there exists a $(d-2)$-flat that stabs at least $c_{d, d-2} n^{3}-O\left(n^{2}\right)$ of the triangles spanned by $S$, with $c_{d, d-2} \geq \frac{1}{24}(1-$ $\left.1 /(2 d-1)^{2}\right)$. To this end, we establish an equipartition result of independent interest (generalizing planar results of Buck and Buck and of Ceder): Every mass distribution
\end{abstract}

Work by B. Bukh was partially carried out at Tel Aviv University.

Work by G. Nivasch was supported by ISF Grant 155/05 and by the Hermann

Minkowski-MINERVA Center for Geometry at Tel Aviv University.

B. Bukh $(\bowtie)$

Department of Mathematics, Fine Hall, Washington Rd., Princeton, NJ 08544, USA

e-mail: bbukh@math.princeton.edu

J. Matoušek

Department of Applied Mathematics and Institute of Theoretical Computer Science (ITI), Charles

University, Malostranské nám. 25, 11800 Prague 1, Czech Republic

e-mail: matousek@kam.mff.cuni.cz

G. Nivasch

Blavatnik School of Computer Science, Tel Aviv University, Tel Aviv 69978, Israel

e-mail: gabriel.nivasch@cs.tau.ac.il 
in $\mathbb{R}^{d}$ can be divided into $4 d-2$ equal parts by $2 d-1$ hyperplanes intersecting in a common $(d-2)$-flat.

Keywords Centerpoint · Equipartition · Equivariant map · Selection lemma · Simplex $\cdot$ Cohomological index

\section{Introduction}

Let $S$ be an $n$-point set in $\mathbb{R}^{d}$ in general position (no $d+1$ points lying on a common hyperplane). The points of $S$ span $\left(\begin{array}{c}n \\ d+1\end{array}\right)$ distinct $d$-dimensional simplices. The following interesting and useful result in discrete geometry (called the First Selection Lemma in [16]) shows that at least a fixed fraction of these simplices have a point in common:

Theorem 1.1 (Bárány [2]) For every $n$-point set $S$ in $\mathbb{R}^{d}$ in general position, there exists a point $p \in \mathbb{R}^{d}$ that is contained in at least $c_{d} n^{d+1}-O\left(n^{d}\right)$ simplices spanned by $S$, where $c_{d}$ is a positive constant depending only on $d$ (and the implicit constant in the $O($ ) notation may also depend on $d$ ).

In this paper we investigate the value of $c_{d}$. More precisely, from now on, let $c_{d}$ denote the supremum of the numbers such that the statement of Theorem 1.1 holds for all finite sets $S$ in $\mathbb{R}^{d}$.

Lower Bounds Bárány's proof yields

$$
c_{d} \geq \frac{1}{d !(d+1)^{d+1}} .
$$

Wagner [22] improved this bound by roughly a factor of $d$, to

$$
c_{d} \geq \frac{d^{2}+1}{(d+1) !(d+1)^{d+1}} .
$$

For the special case $d=2$, Boros and Füredi [7] achieved the better lower bound of $c_{2}=1 / 27$ (also see Bukh [8] for a simpler proof of this planar bound).

Upper Bounds The following result was proved by Kárteszi [15] for $d=2$ (also see Moon [18, p. 7] and Boros and Füredi [6,7]) and by Bárány [2] for general $d$ :

Theorem 1.2 If $S$ is any n-point set in general position in $\mathbb{R}^{d}$, then no point $p \in \mathbb{R}^{d}$ is contained in more than

$$
\frac{1}{2^{d}(d+1) !} n^{d+1}+O\left(n^{d}\right)
$$

$d$-simplices spanned by $S$. 
It follows without difficulty from a result of Wendel (reproduced as Lemma 3.1 below) that this bound is asymptotically attained with high probability by points chosen uniformly at random from the unit sphere. Alternatively, as was kindly pointed out to us by Uli Wagner, the tightness also follows by considering the Gale transform of the polar of a cyclic polytope; see, e.g., Welzl [23] for the relevant background.

Bárány's bound implies that

$$
c_{d} \leq \frac{1}{2^{d}(d+1) !},
$$

which, to our knowledge, was the best known upper bound on $c_{d}$ for all $d \geq 3$.

For $d=2$, Boros and Füredi [7] claimed the upper bound $c_{2} \leq 1 / 27$ (which would be tight), but it turns out that the construction in their paper gives only $c_{2} \leq 1 / 27+$ 1/729 (see Appendix of this paper).

Our first result is an improved upper bound for $c_{d}$ for every $d$ (and the first "nontrivial" one, in the sense that it refers to a specific construction):

Theorem 1.3 For every fixed $d \geq 2$ and every $n$, there exists an $n$-point set $S \subset \mathbb{R}^{d}$ such that no point $p \in \mathbb{R}^{d}$ is contained in more than $(n /(d+1))^{d+1}+O\left(n^{d}\right) d$ simplices spanned by $S$. Thus,

$$
c_{d} \leq(d+1)^{-(d+1)} .
$$

Moreover, such an $S$ can be chosen in convex position.

In particular, the planar bound of $c_{2}=1 / 27$ is tight, after all.

\subsection{The First Selection Lemma and Centerpoints}

If $S$ is an $n$-point set in $\mathbb{R}^{d}$ and $p \in \mathbb{R}^{d}$, we say that $p$ lies at depth $m$ with respect to $S$ if every halfspace that contains $p$ contains at least $m$ points of $S$. A classical result of Rado [20] states that there always exists a point at depth $n /(d+1)$. Such a point is called a centerpoint.

Wagner proved the bound (1) by showing the following:

Theorem 1.4 [22] If $S$ is an n-point set in $\mathbb{R}^{d}$ and $p \in \mathbb{R}^{d}$ is a point at depth $\alpha n$ with respect to $S$, then $p$ is contained in at least

$$
\left((d+1) \alpha^{d}-2 d \alpha^{d+1}\right) \frac{n^{d+1}}{(d+1) !}-O\left(n^{d}\right)
$$

$d$-simplices spanned by $S .^{1}$

This, together with Rado's Centerpoint Theorem, immediately implies (1).

In this paper we show that Theorem 1.4 cannot be improved:

\footnotetext{
${ }^{1}$ This is obtained by setting $k=0$ in the lower bound for $f_{k}(\mu, \mathbf{o})$ in the proof of Theorem 4.32 in [22].
} 
Theorem 1.5 For every $\alpha, 0<\alpha \leq 1 / 2$, and every $n$, there exists an $n$-point set $S$ in $\mathbb{R}^{d}$ such that the origin is at depth $\alpha n$ with respect to $S$ but is contained in only

$$
\left((d+1) \alpha^{d}-2 d \alpha^{d+1}\right) \frac{n^{d+1}}{(d+1) !}+O\left(n^{d}\right)
$$

$d$-simplices spanned by $S$.

Thus, the approach of taking an arbitrary centerpoint cannot yield any lower bound better than (1) for the First Selection Lemma.

\subsection{Stabbing $(d-k)$-simplices by $k$-flats}

The First Selection Lemma can be generalized as follows: If $S$ is an $n$-point set in $\mathbb{R}^{d}$ and $k$ is an integer, $0 \leq k<d$, then there exists a $k$-flat that intersects at least $c_{d, k} n^{d-k+1}-O\left(n^{d-k}\right)$ of the $(d-k)$-simplices spanned by $S$, for some positive constants $c_{d, k}$ that depend only on $d$ and $k$. (By a $k$-flat we mean a $k$-dimensional affine subspace of $\mathbb{R}^{d}$.)

The problem is to determine the maximum values of the constants $c_{d, k}$. Trivially we have $c_{d, k} \geq c_{d-k}$ : Simply project $S$ into an arbitrary generic subspace of dimension $d-k$ and then apply the First Selection Lemma.

Here we derive a nontrivial lower bound for the case $k=d-2$ (this is the only case for which we could obtain good lower bounds):

Theorem 1.6 If $S$ is an n-point set in $\mathbb{R}^{d}$, then there is a $(d-2)$-flat $\ell$ that intersects at least $\frac{1}{24}\left(1-1 /(2 d-1)^{2}\right) n^{3}-O\left(n^{2}\right)$ triangles spanned by $S$. Thus,

$$
c_{d, d-2} \geq \frac{1}{24}\left(1-\frac{1}{(2 d-1)^{2}}\right) .
$$

For $d=2$, this is just the planar version of First Selection Lemma with the optimal constant $1 / 27$. Moreover, as $d$ increases, the right-hand-side of (3) increases strictly with $d$, approaching $1 / 24$ as $d$ tends to infinity. Indeed, it is impossible to stab more than $n^{3} / 24$ triangles for any $d$, since then projecting into a plane perpendicular to $\ell$ would result in a point stabbing more than $n^{3} / 24$ triangles in the plane, contradicting Theorem 1.2.

Theorem 1.6 is a consequence of the following equipartition result, which is interesting in its own right. Given an integer $m \geq 2$, define an $m$-fan as a set of $m$ hyperplanes in $\mathbb{R}^{d}$ that pass through a common $(d-2)$-flat. Then:

Theorem 1.7 For every probability measure $\mu$ on $\mathbb{R}^{d}$ that is absolutely continuous with respect to the Lebesgue measure, there exists a $(2 d-1)$-fan that divides $\mu$ into $4 d-2$ equal parts.

For $d=2$, this theorem specializes to a result of Ceder [9] (also see Buck and Buck [5] for a special case).

We also show that $2 d-1$ is the largest possible number of hyperplanes in Theorem 1.7: 
Theorem 1.8 For every integer $m \geq 2 d$, there exists an absolutely continuous probability measure $\mu$ on $\mathbb{R}^{d}$ that cannot be partitioned into $2 m$ equal parts by an $m$-fan.

\section{The Construction for Theorem 1.3}

We now prove Theorem 1.3 by constructing a suitable point set $S$. Given real numbers $a, b>1$, let $a \ll b$ mean that $f(a)<b$ for some fixed, sufficiently large function $f$ (concretely, we can take $\left.f(x)=(d+1) ! x^{d+1}\right)$. Our point set is $S=\left\{p_{1}, \ldots, p_{n}\right\}$ with

$$
p_{i}=\left(p_{i 1}, p_{i 2}, \ldots, p_{i d}\right) \in(1, \infty)^{d},
$$

where the components $p_{i j}$ satisfy

$$
p_{i j} \ll p_{i^{\prime} j^{\prime}} \quad \text { whenever } j<j^{\prime} \text {, or } j=j^{\prime} \text { and } i<i^{\prime}
$$

(so the ordering of the $p_{i j}$ is first by the coordinate index $j$ and then by the point index $i$ ). The idea of taking points separated by rapidly increasing distances is borrowed from Boros and Füredi's planar construction [7]. However, their construction is more complicated, with points grouped into three clusters; see Appendix.

Lemma 2.1 The set $S$ is in convex position.

Proof Let $p_{i}^{\prime}=\left(p_{i 1}, p_{i 2}\right)$ be the projection of the point $p_{i}$ into the $x_{1} x_{2}$-plane for $1 \leq i \leq n$. We claim that the points $p_{i}^{\prime}$ lie on an $x_{1}$-monotone convex curve in the $x_{1} x_{2}$-plane (which implies the lemma).

To this end, we show that for every three points $p_{i}^{\prime}, p_{j}^{\prime}, p_{k}^{\prime}$ with $i<j<k$, the segment $p_{i}^{\prime} p_{j}^{\prime}$ has a smaller slope than the segment $p_{j}^{\prime} p_{k}^{\prime}$; see Fig. 1(a). Indeed, this is the case if and only if

$$
\left(p_{k 2}-p_{j 2}\right)\left(p_{j 1}-p_{i 1}\right)>\left(p_{j 2}-p_{i 2}\right)\left(p_{k 1}-p_{j 1}\right) .
$$

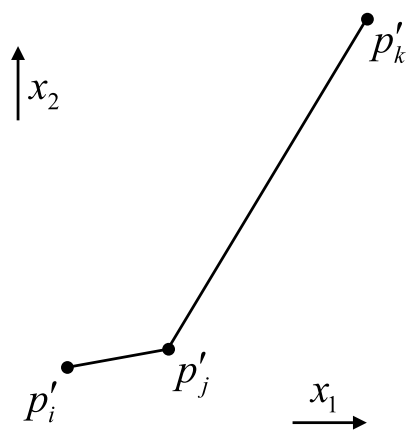

(a)

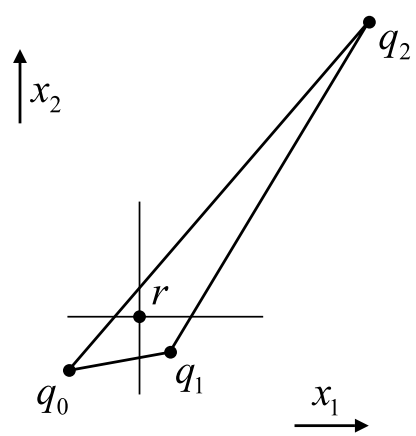

(b)

Fig. 1 (a) In the $x_{1} x_{2}$-plane, the segment $p_{i}^{\prime} p_{j}^{\prime}$ has a smaller slope than the segment $p_{j}^{\prime} p_{k}^{\prime}$. (b) In the planar case, the point $r$ must lie above-right of $q_{0}$, above-left of $q_{1}$, and below-left of $q_{2}$ 
However, (4) holds as long as the function $f$ in the definition of $\ll$ is chosen large enough. Specifically, if $f(x) \geq 4 x^{2}$, then the left-hand side of (4) is at least

$$
\frac{1}{2} p_{k 2} \cdot \frac{1}{2} p_{j 1} \geq \frac{1}{4} p_{k 2} \geq p_{j 2}^{2},
$$

which is larger than the right-hand side of (4).

Next, we want to show that no point $r=\left(r_{1}, \ldots, r_{d}\right) \in \mathbb{R}^{d}$ is contained in more than $(n /(d+1))^{d+1}+O\left(n^{d}\right)$ of the $d$-simplices spanned by $S$.

We can assume that $p_{1 j} \leq r_{j} \leq p_{n j}$ for each coordinate $1 \leq j \leq d$, since otherwise, $r$ is not contained in any $d$-simplex spanned by $S$. For each coordinate $j=1, \ldots, d$, we discard from $S$ the last point $p_{i}$ with $p_{i j} \leq r_{j}$ and the first point $p_{i}$ with $p_{i j} \geq r_{j}$. Let $S^{\prime}$ be the resulting set. Since we have discarded at most $2 d$ points, the number of $d$-simplices involving any of the discarded points is only $O\left(n^{d}\right)$. And now, for every $p_{i} \in S^{\prime}$ and every $j$, we have either $r_{j} \ll p_{i j}$ or $r_{j} \gg p_{i j}$.

Let $a=\left(a_{1}, \ldots, a_{d}\right) \in \mathbb{R}^{d}$ be a point; we define the type of $a$ with respect to $r$ as $\max \left\{k: a_{j}>r_{j}\right.$ for all $\left.j=1,2, \ldots, k\right\}$. Note that the type of $a$ is an integer between 0 and $d$ (it is 0 if $a_{1} \leq r_{1}$ ).

Let $p_{i_{0}}, \ldots, p_{i_{d}} \in S^{\prime}$ span a $d$-simplex containing $r$, with $i_{0}<\cdots<i_{d}$. For convenience, we rename these points and their coordinates as

$$
q_{\ell}=\left(q_{\ell 1}, \ldots, q_{\ell d}\right) \text { for } \ell=0,1, \ldots, d .
$$

The proof of Theorem 1.3 will be almost finished once we establish the following:

Lemma 2.2 For each $\ell=0,1, \ldots, d$, the point $q_{\ell}$ has type $\ell$ with respect to $r$. (See Fig. 1(b) for an illustration of the planar case.)

Indeed, assuming this lemma, the proof of Theorem 1.3 is concluded as follows. Given $r$, we partition the points of $S^{\prime}$ into $d+1$ subsets $S_{0}^{\prime}, \ldots, S_{d}^{\prime}$ according to their type. Then, for a $d$-simplex spanned by $d+1$ points from $S^{\prime}$ to contain $r$, each point must come from a different $S_{k}^{\prime}$. The number of such simplices is thus at most $\prod_{k=0}^{d}\left|S_{k}^{\prime}\right| \leq(n /(d+1))^{d+1}$, by the arithmetic-geometric mean inequality.

Proof of Lemma 2.2 We are going to derive the following relations:

$$
q_{(j-1) j} \ll r_{j} \ll q_{j j} \quad \text { for every } j=1,2, \ldots, d \text {. }
$$

Let us first check that they imply the lemma. To see that $q_{\ell}$ has type $\ell$, we need that $q_{\ell j}>r_{j}$ for $j \leq \ell$ and, if $\ell<d$, also that $q_{\ell(\ell+1)} \leq r_{\ell+1}$. The last inequality follows from (5) with $j=\ell+1$. To derive $q_{\ell j}>r_{j}$, note that the coordinates of $q_{\ell}$ are increasing since $q_{\ell} \in S$, and thus $q_{\ell j} \geq q_{j j}>r_{j}$.

Now we start working on (5). First we express the condition that $r$ lies in the simplex spanned by $q_{0}, \ldots, q_{d}$ using determinants. For each $\ell$, the points $r$ and $q_{\ell}$ must lie on the same side of the hyperplane spanned by the points $q_{m}, m \neq \ell$. Thus, let $M$ be the $(d+1) \times(d+1)$ matrix consisting of the rows $\left(1, q_{0}\right),\left(1, q_{1}\right), \ldots,\left(1, q_{d}\right)$. 
For $k=0,1, \ldots, d$, let $M_{k}$ be the matrix obtained from $M$ by replacing the row $\left(1, q_{k}\right)$ by $(1, r)$. Then, for each $k, \operatorname{det} M_{k}$ must have the same sign as $\operatorname{det} M$.

Next, we show that $\operatorname{det} M$ and each $\operatorname{det} M_{k}$ are "dominated" by a single product of entries. Let $A$ be one of the matrices $M, M_{0}, M_{1}, \ldots, M_{d}$, and denote by $a_{\ell j}$ the entry in row $\ell$ and column $j$ of $A$ for $0 \leq \ell, j \leq d$. We claim that if the function $f$ in the definition of $\ll$ is chosen sufficiently large, then there is a single product of the form $\operatorname{sign}(\sigma) \prod_{\ell} a_{\ell \sigma(\ell)}$, for some permutation $\sigma$, which is larger in absolute value than the sum of absolute values of all the other products in $\operatorname{det} A$.

Indeed, let $a_{\ell_{d} d}$ be the largest entry in the last column of $A$. This is also the largest entry in the entire matrix. Then, if we take $f(x) \geq(d+1) ! x^{d+1}$, any permutation product involving $a_{l_{d}}$ is larger than $(d+1)$ ! times any permutation product not involving this entry. Thus, we choose $a_{\ell_{d} d}$ as the first term in our product, we remove row $\ell_{d}$ and column $d$ from $A$, and we continue in this way leftwards. We obtain a product $\prod_{\ell} a_{\ell \sigma(\ell)}$ which is larger than $(d+1)$ ! times any other permutation product in $\operatorname{det} A$. Therefore, this product "dominates" $\operatorname{det} A$ in the above sense, and so $\operatorname{sign}(\operatorname{det} A)=\operatorname{sign}(\sigma)$.

In particular, these considerations for $A=M$ show that $\operatorname{det} M$ is dominated by the product

$$
q_{d d} q_{(d-1)(d-1)} \cdots q_{11} \cdot 1
$$

corresponding to the identity permutation. Therefore, $\operatorname{det} M>0$, and so we must have $\operatorname{det} M_{k}>0$ for all $k$.

Now we are ready to prove (5). First we suppose for contradiction that $r_{j} \gg q_{j j}$ for some $j=1,2, \ldots, d$. We take the largest such $j$; thus, $q_{k k} \gg r_{k}$ for $k>j$. Then $\operatorname{det} M_{j-1}$ is dominated by the product

$$
q_{d d} \cdots q_{(j+1)(j+1)} r_{j} q_{j(j-1)} q_{(j-2)(j-2)} \cdots q_{11} \cdot 1,
$$

so the sign of det $M_{j-1}$ is the sign of the permutation associated with this product. This is a permutation with exactly one inversion, so $\operatorname{det} M_{j-1}<0$, which is a contradiction.

Next, we suppose for contradiction that $r_{j} \ll q_{(j-1) j}$ for some $j=1,2, \ldots, d$. Now we take the smallest such $j$. We have already shown that $r_{k} \ll q_{k k}$ for all $k$. Therefore, $\operatorname{det} M_{j}$ is dominated by the product

$$
q_{d d} \cdots q_{(j+1)(j+1)} q_{(j-1) j} r_{j-1} q_{(j-2)(j-2)} \cdots q_{11} \cdot 1 .
$$

Again, this product corresponds to a permutation with exactly one inversion, so we have $\operatorname{det} M_{j}<0$, which is again a contradiction.

\section{The Construction for Theorem 1.5}

We now present the construction that proves Theorem 1.5. Let us call a set $Y \subseteq \mathbb{R}^{d}$ antisymmetric if $Y \cap(-Y)=\emptyset$.

We make use of the following result of Wendel: 
Lemma 3.1 [24] Let $X=\left\{x_{1}, \ldots, x_{d+1}\right\}$ be a set of $d+1$ points in general position on the unit sphere $\mathbb{S}^{d-1}$ in $\mathbb{R}^{d}$. Then there are exactly two antisymmetric $(d+1)$-point subsets of $X \cup(-X)$ whose convex hull contains the origin; if one of them is $Y$, then the other one is $-Y$.

Let $\alpha \in(0,1 / 2]$ be a parameter, and let $n$ be given. For the moment, assume for simplicity that $\alpha n$ is an integer. Let $A$ be a set of $\alpha n$ points on $\mathbb{S}^{d-1}$, and let $p$ be another point on $\mathbb{S}^{d-1}$ such that $A \cup\{p\}$ is in general position-namely, such that the set $A \cup(-A) \cup\{p\}$ has $2|A|+1$ points and no hyperplane containing $p$ and a $(d-1)$-point antisymmetric subset of $A \cup(-A)$ passes through the origin. Let $P$ be a very small cluster of $(1-2 \alpha) n$ points around $p$.

Our set is $S=A \cup(-A) \cup P$. Note that $|S|=n$ as required. The origin clearly lies at depth $\alpha n$ with respect to $S$. Thus, Theorem 1.5 reduces to the following lemma:

Lemma 3.2 The number of $(d+1)$-point subsets $B$ of $S$ such that conv $B$ contains the origin is

$$
\left((d+1) \alpha^{d}-2 d \alpha^{d+1}\right)\left(\begin{array}{c}
n \\
d+1
\end{array}\right)+O\left(n^{d}\right)
$$

Proof The number of $(d+1)$-point subsets of $S$ that contain a pair of antipodal points (one in $A$ and one in $-A$ ) is $O\left(n^{d}\right)$, and so it suffices to count the number of $B$ that are antisymmetric.

The choice of $A$ and $P$ guarantees that if $B$ is antisymmetric and $|B \cap P| \geq 2$, then $0 \notin \operatorname{conv} B$. So we need to consider the cases $B \cap P=\emptyset$ and $|B \cap P|=1$.

Let us set $\tilde{B}=\{x \in A \cup P: x \in B$ or $-x \in B\}$. For $B \cap P=\emptyset$, there are $\left(\begin{array}{c}\alpha n \\ d+1\end{array}\right)$ ways of choosing $\tilde{B} \subseteq A$, and for each of them, we have two choices for $B$ by Lemma 3.1.

For $|B \cap P|=1$, we have $(1-2 \alpha) n\left(\begin{array}{c}\alpha n \\ d\end{array}\right)$ choices for $\tilde{B}$, and each of them yields exactly one $B$ (Lemma 3.1 with $X=\tilde{B}$ shows that there are two $B \subset \tilde{B} \cup(-\tilde{B})$ with $0 \in \operatorname{conv} B$, and exactly one of these contains the point $p \in P \cap \tilde{B}$, while the other contains $-p$ ).

Altogether, the number of $B$ 's is $2\left(\begin{array}{c}\alpha n \\ d+1\end{array}\right)+(1-2 \alpha) n\left(\begin{array}{c}\alpha n \\ d\end{array}\right)+O\left(n^{d}\right)$, and the lemma follows by algebraic manipulation.

If $\alpha n$ is not an integer, then apply the above argument using $\alpha^{\prime}=\lceil\alpha n\rceil / n$ and use the fact that $\alpha^{\prime}-\alpha<1 / n$.

\section{Partitioning Measures by Fans of Hyperplanes}

In this section we prove Theorem 1.7, which is the main ingredient in the proof of Theorem 1.6. We then prove Theorem 1.8, showing that Theorem 1.7 is optimal. Recall that an $m$-fan is a set of $m$ hyperplanes in $\mathbb{R}^{d}$ sharing a common $(d-2)$-flat.

Let $\mathbb{S}^{d-1}$ denote the unit sphere in $\mathbb{R}^{d}$, and let $\mathbb{V}_{d, 2}=\left\{(v, w) \in\left(\mathbb{S}^{d-1}\right)^{2}: v \perp w\right\}$ denote the set of ordered pairs of orthonormal vectors in $\mathbb{R}^{d}$ (called the Stiefel manifold of orthogonal 2-frames). The proof of Theorem 1.7 is based on the following topological result: 
Lemma 4.1 There exists no continuous function $g: \mathbb{V}_{d, 2} \rightarrow \mathbb{S}^{2 d-4}$ with the following property: For every $(v, w) \in \mathbb{V}_{d, 2}$, if $g(v, w)=\left(a_{1}, \ldots, a_{2 d-3}\right)$ (with $a_{1}^{2}+\cdots+$ $\left.a_{2 d-3}^{2}=1\right)$, then

- $g(-v, w)=\left(-a_{1}, \ldots,-a_{d-1}, a_{d}, \ldots, a_{2 d-3}\right)$, and

- $g(v,-w)=\left(a_{1}, \ldots, a_{d-1},-a_{d}, \ldots,-a_{2 d-3}\right)$.

Proof The lemma is a result on nonexistence of an equivariant map. Let us briefly recall the basic setting; for more background, we refer to [17, 26].

Let $G$ be a finite group. A $G$-space is a topological space $X$ together with an action of $G$ on $X$, which is a collection $\left(\varphi_{g}\right)_{g \in G}$ of homeomorphisms $\varphi_{g}: X \rightarrow X$ whose composition agrees with the group operation in $G$; that is, $\varphi_{e}=\operatorname{id}_{X}$ for the unit element $e \in G$ and $\varphi_{g} \circ \varphi_{h}=\varphi_{g h}$ for all $g, h \in G$.

In our case, the relevant group is $G:=\mathbb{Z}_{2} \times \mathbb{Z}_{2}$ (the direct product of two cyclic groups of order 2). We can write $G=\left\{e, g_{1}, g_{2}, g_{1} g_{2}\right\}$, where $g_{1}$ and $g_{2}$ are two generators of $G$; in order to specify an action of $G$, it is enough to give the homeomorphisms corresponding to $g_{1}$ and $g_{2}$. The lemma deals with two $G$-spaces:

- The Stiefel manifold $\mathbb{V}_{d, 2}$ with the action $\left(\varphi_{g}\right)_{g \in G}$ of $G$ given by $\varphi_{g_{1}}(v, w)=$ $(-v, w), \varphi_{g_{2}}(v, w)=(v,-w)$.

- The sphere $\mathbb{S}^{2 d-4}$ with the action $\left(\psi_{g}\right)_{g \in G}$, where $\psi_{g_{1}}$ flips the signs of the first $d-1$ coordinates, and $\psi_{g_{2}}$ flips the signs of the remaining $d-2$ coordinates.

We want to prove that there is no equivariant map $f: \mathbb{V}_{d, 2} \rightarrow \mathbb{S}^{2 d-4}$, where an equivariant map is a continuous map that commutes with the actions of $G$, i.e., such that $f \circ \varphi_{g}=\psi_{g} \circ f$ for all $g \in G$. The "usual" elementary methods for showing nonexistence of equivariant maps, explained in $[17,26]$ and based on the BorsukUlam theorem and its generalizations, cannot be applied here. We use the idealvalued cohomological index of Fadell and Husseini [13] (also see [25]).

This method assigns to every $G$-space $X$ the $G$-index of $X$, denoted by $\operatorname{Ind}_{G}(X)$, which is an ideal in a certain ring $R_{G}$ (depending only on $G$ ). A key property is that whenever there is an equivariant map $f: X \rightarrow Y$, where $X$ and $Y$ are $G$-spaces, we have $\operatorname{Ind}_{G}(Y) \subseteq \operatorname{Ind}_{G}(X)$. For the considered $G=\mathbb{Z}_{2} \times \mathbb{Z}_{2}, R_{G}$ is the ring $\mathbb{Z}_{2}\left[t_{1}, t_{2}\right]$ of polynomials in two variables with $\mathbb{Z}_{2}$ coefficients. The general definition of $\operatorname{Ind}_{G}(X)$, as well as its computation, are rather complicated, but fortunately, in our case we can use ready-made results from the literature.

For the $G$-space $\mathbb{S}^{2 d-4}$ with the $G$-action as above, the $G$-index is the principal ideal in $\mathbb{Z}_{2}\left[t_{1}, t_{2}\right]$ generated by $t_{1}^{d-1} t_{2}^{d-2}$ according to Corollary 2.12 in [25]. On the other hand, Fadell [12] proved that the $G$-index of the $G$-space $\mathbb{V}_{d, 2}$ with the described $G$-action does not contain the monomial $t_{1}^{d-1} t_{2}^{d-2}$ (also see [14] for a statement of this result and some applications of it). This shows that an equivariant map as in the lemma is indeed impossible.

Proof of Theorem 1.7 We follow the "configuration space/test map" paradigm (see, e.g., [26]). We encode each "candidate" for the desired equipartition, which in our case is going to be a certain special fan of $4 d-2$ half-hyperplanes sharing the boundary $(d-2)$-flat, by a point of $\mathbb{V}_{d, 2}$. Then we define a continuous test map that assigns to each candidate fan of half-hyperplanes a $(2 d-3)$-tuple of real numbers, which 
Fig. $22 m$ half-hyperplanes coming out of $\ell$ that partition the measure $\mu$ into $2 m$ equal parts. (Here $m=5$ )

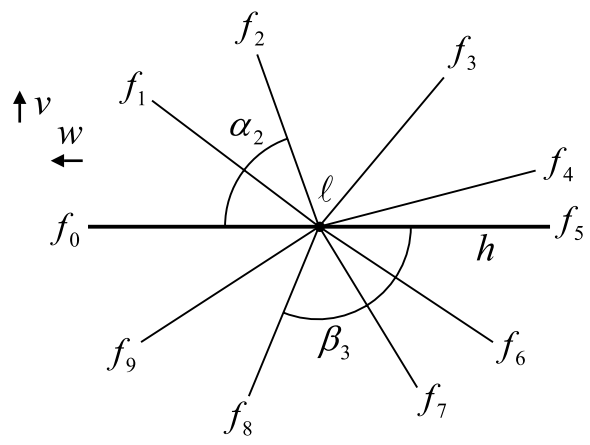

measures how far the given candidate is from being a $(2 d-1)$-fan of hyperplanes. Finally we will check that if there were no equipartition, the test map would yield an equivariant map $\mathbb{V}_{d, 2} \rightarrow \mathbb{S}^{2 d-4}$, which would contradict Lemma 4.1. The details follow.

For the proof, we may assume that every nonempty open set has a positive $\mu$ measure. (Given an arbitrary $\mu$, we can consider the convolution $\mu * \gamma_{\varepsilon}$ of $\mu$ with a suitable probability measure $\gamma_{\varepsilon}$ whose density function is everywhere nonzero but for which all but at most $\varepsilon$ of the mass lies in a ball of radius $\varepsilon$ around 0 . The convolution has the required property and then, given an equipartition for each $\mu * \gamma_{\varepsilon}$, a limit argument, letting $\varepsilon \rightarrow 0$, yields an equipartition for the original $\mu$. See the proof of [17, Theorem 3.1.1] for a similar limit argument.)

Let $m=2 d-1$. Suppose that we are given two orthonormal vectors $v, w \in \mathbb{S}^{d-1}$. Let $h$ be the unique hyperplane orthogonal to $v$ that splits $\mathbb{R}^{d}$ into two halfspaces of equal measure with respect to $\mu$. We say that the halfspace in the direction of $v$ is "above" $h$, and the other halfspace is "below" $h$.

Let $\ell$ be a $(d-2)$-flat orthogonal to $w$ contained in $h$. Note that $\ell$ splits $h$ into two half-hyperplanes. We say that the half of $h$ in the direction of $w$ lies "left" of $\ell$, and the other half of $h$ lies "right" of $\ell$.

Every half-hyperplane with boundary $\ell$ is uniquely determined by the angle it makes with the left half of $h$. Let $f_{0}, f_{1}, \ldots, f_{2 m-1}$ be $2 m$ half-hyperplanes coming out of $\ell$, listed in circular order, that split the measure $\mu$ into $2 m$ equal parts, as follows:

- $f_{0}$ is the left half of $h$

- $f_{1}, \ldots, f_{m-1}$ lie above $h$

- $f_{m}$ is the right half of $h$, and

- $f_{m+1}, \ldots, f_{2 m-1}$ lie below $h$.

See Fig. 2.

For $i=1, \ldots, m-1$, let $\alpha_{i}$ be the angle between $f_{0}$ and $f_{i}$, and let $\beta_{i}$ be the angle between $f_{m}$ and $f_{m+i}$. Let $\gamma_{i}=\alpha_{i}-\beta_{i}$. Note that $\gamma_{i}=0$ means that $f_{i}$ and $f_{m+i}$ are aligned into a hyperplane.

Translating $\ell$ within $h$ to the left causes the $\alpha_{i}$ 's to increase and the $\beta_{i}$ 's to decrease, while translating it to the right has the opposite effect. Therefore, there exists a unique position of $\ell$ for which $\sum \alpha_{i}=\sum \beta_{i}$, or equivalently, $\sum \gamma_{i}=0$, and we fix 

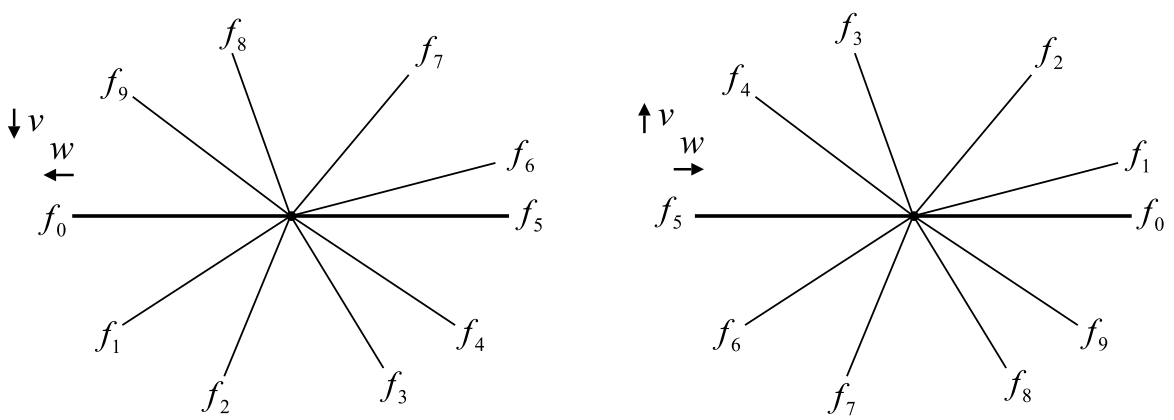

Fig. 3 The effect of changing the sign of $v$ (left) or $w$ (right)

$\ell$ there. In this way, we have defined each $\alpha_{i}$ and $\beta_{i}$ as a function of the given vectors $v, w$. Using the assumption that $\mu$ is absolutely continuous with respect to the Lebesgue measure and each open set has a positive $\mu$-measure, it is routine to verify the continuity of the $\alpha_{i}$ and $\beta_{i}$ as functions of $v$ and $w$.

Let us examine what happens when we change the sign of $v$ or $w$. We have

$$
\begin{array}{ll}
\alpha_{i}(-v, w)=\pi-\beta_{m-i}(v, w), & \beta_{i}(-v, w)=\pi-\alpha_{m-i}(v, w), \\
\alpha_{i}(v,-w)=\pi-\alpha_{m-i}(v, w), & \beta_{i}(v,-w)=\pi-\beta_{m-i}(v, w) .
\end{array}
$$

See Fig. 3. Therefore,

$$
\begin{aligned}
& \gamma_{i}(-v, w)=\gamma_{m-i}(v, w), \\
& \gamma_{i}(v,-w)=-\gamma_{m-i}(v, w) .
\end{aligned}
$$

Now we introduce a suitable change of coordinates in the target space so that the resulting map behaves as the map $g$ considered in Lemma 4.1. Namely, we set

$$
\begin{array}{ll}
\lambda_{i}=\gamma_{i}-\gamma_{m-i} & \text { for } i=1, \ldots,(m-1) / 2, \\
\mu_{i}=\gamma_{i}+\gamma_{m-i} & \text { for } i=2, \ldots,(m-1) / 2 .
\end{array}
$$

Note that

$$
\begin{array}{lr}
\lambda_{i}(-v, w)=-\lambda_{i}(v, w), & \mu_{i}(-v, w)=\mu_{i}(v, w), \\
\lambda_{i}(v,-w)=\lambda_{i}(v, w), & \mu_{i}(v,-w)=-\mu_{i}(v, w) .
\end{array}
$$

We have $\gamma_{i}=0$ for all $i$ if and only if $\lambda_{i}, \mu_{i}=0$ for all $i$. (Recall that $\sum \gamma_{i}=0$.) Now we define the "test map" $G: \mathbb{V}_{d, 2} \rightarrow \mathbb{R}^{m-2}$ by

$$
G(v, w)=\left(\lambda_{1}, \ldots, \lambda_{(m-1) / 2}, \mu_{2}, \ldots, \mu_{(m-1) / 2}\right) .
$$

Then, our desired equipartition of $\mu$ exists if and only if $G(v, w)=(0, \ldots, 0)$ for some $(v, w)$. 
However, $G$ is a continuous map such that flipping $v$ flips the first $(m-1) / 2=$ $d-1$ coordinates of the image, while flipping $w$ flips the last $(m-3) / 2=d-2$ coordinates of the image. If we had $G \neq(0, \ldots, 0)$ for all $(v, w)$, the map $g: \mathbb{V}_{d, 2} \rightarrow$ $\mathbb{S}^{2 d-4}$ given by $g(v, w)=G(v, w) /\|G(v, w)\|$ would contradict Lemma 4.1. Therefore, the desired equipartition exists.

We conclude this section by proving Theorem 1.8, which shows that Theorem 1.7 is best possible, in the sense that an equipartition of a measure $\mu$ in $\mathbb{R}^{d}$ by a fan of $2 d$ or more hyperplanes does not necessarily exist. The proof is based on the following lemma:

Lemma 4.2 Let $m>0$ be an integer, and let $t \geq 2 d+m-1$. Then there exists $a$ $t$-point set $T \subset \mathbb{R}^{d}$ that cannot be covered by any $m$-fan in $\mathbb{R}^{d}$.

The basic idea, roughly speaking, is that an $m$-fan in $\mathbb{R}^{d}$ has $2 d+m-2$ degrees of freedom, while each point in $T$ takes away one degree of freedom. Therefore, $T$ can be completely covered by an $m$-fan only if it is degenerate an appropriate sense.

Proof of Lemma 4.2 For convenience, we first prove the result in $\mathbb{R P}^{d}$, the $d$ dimensional projective space, and then we show that the result also applies to $\mathbb{R}^{d}$.

A set of $m$ hyperplanes in $\mathbb{R P}^{d}$ share a common $(d-2)$-flat if and only if their dual points, when considered as vectors in $\mathbb{R}^{d+1}$, span a vector space of dimension at most 2 . Thus, define the projective variety

$$
V=\left\{\left(p_{1}, \ldots, p_{m}\right) \in\left(\mathbb{R P}^{d}\right)^{m}: \operatorname{rank}\left(p_{1}, \ldots, p_{m}\right) \leq 2\right\},
$$

where $\operatorname{rank}\left(p_{1}, \ldots, p_{m}\right)$ denotes the dimension of the vector space spanned by $p_{1}, \ldots, p_{m}$ as vectors in $\mathbb{R}^{d+1}$. The variety $V$ has dimension $\operatorname{dim} V=2 d+m-2$.

Given a point $p=p_{0}: p_{1}: \cdots: p_{d} \in \mathbb{R P}^{d}$, let $p^{*}=\left\{x_{0}: \cdots: x_{d} \in \mathbb{R P}^{d}\right.$ : $\left.\sum_{i} x_{i} p_{i}=0\right\}$ denote the hyperplane dual to $p$. For each $v=\left(p_{1}, \ldots, p_{m}\right) \in V$, let $v^{*}=\bigcup_{i} p_{i}^{*} \subset \mathbb{R} \mathbb{P}^{d}$ be the variety which consists of the union of the hyperplanes dual to the points in $v$. Finally, define the moduli space

$$
C=\left\{\left(v, q_{1}, \ldots, q_{t}\right) \in V \times\left(\mathbb{R P}^{d}\right)^{t}: q_{1}, \ldots, q_{t} \in v^{*}\right\}
$$

of all $t$-tuples of points lying on a fan $v^{*}$, for all $v \in V$. The dimension of $C$ is $\operatorname{dim} C=\operatorname{dim} V+t(d-1)=2 d+m-2+t(d-1)$.

Consider the projection map $\pi: V \times\left(\mathbb{R P}^{d}\right)^{t} \rightarrow\left(\mathbb{R P}^{d}\right)^{t}$. Then the projection $\pi(C)$ is the set of $t$-tuples of points in $\mathbb{R P}^{d}$ that can be covered by an $m$-fan. By the TarskiSeidenberg Theorem [4] $\pi(C)$ is a semialgebraic subset of $\left(\mathbb{R P}^{d}\right)^{t}$. Since projection does not increase the dimension, $\pi(C)$ is of dimension at most $2 d+m-2+t(d-1)$, which by our choice of $t$ is smaller than $t d=\operatorname{dim}\left(\mathbb{R P}^{d}\right)^{t}$.

Thus, there exists a $t$-point set $T$ in $\mathbb{R P}^{d}$ that cannot be covered by an $m$-fan. Finally, a generic $\mathbb{R}^{d}$ inside $\mathbb{R P}^{d}$ completely contains $T$, and the lemma follows.

Proof of Theorem 1.8 Given an integer $m \geq 2 d$, let $t=2 m-1$. We have $t \geq 2 d+$ $m-1$, so by the preceding lemma there exists a $t$-point set $T \subset \mathbb{R}^{d}$ that cannot be covered by any $m$-fan in $\mathbb{R}^{d}$. 
There must exist a positive radius $r$ such that, for every $m$-fan $F$ in $\mathbb{R}^{d}$, some point of $T$ lies at distance at least $r$ from the closest hyperplane in $F$. (Otherwise a limit argument would yield an $m$-fan that covers $T$.)

Let $C_{r}(p)$ denote the ball of radius $r$ centered at $p$. Let $\mu$ be the uniform measure on $\bigcup_{p \in T} C_{r}(p)$. Then, in every partition of $\mathbb{R}^{d}$ into $2 m$ parts by an $m$-fan, there exists a part that completely contains one of the balls $C_{r}(p)$. This part has measure at least $\frac{1}{t}>1 /(2 m)$, and so the partition is not an equipartition.

\section{Stabbing Many Triangles in $\mathbb{R}^{d}$}

In this section we prove Theorem 1.6 by means of Theorem 1.7. The proof is an extension of the technique in [8] for the case $d=2$.

By a standard approach (see, e.g., Theorem 3.1.2 in [17]), Theorem 1.7 implies the following discrete version, which is what we actually use:

Corollary 5.1 Let $S$ be a set of $n$ points in $\mathbb{R}^{d}$. Then there exist $2 d-1$ hyperplanes passing through a common $(d-2)$-flat that divide the space into $4 d-2$ parts, each containing at most $n /(4 d-2)+O(1)$ points of $S$.

We start with the following lemma:

Lemma 5.2 Let $\ell_{0}, \ldots, \ell_{m-1}$ be $m$ lines in the plane passing through a common point $x$ and dividing the plane into $2 m$ sectors. Let $P=\left\{p_{0}, \ldots, p_{2 m-1}\right\}$ be $2 m$ points, one from each sector, listed in circular order around $x$. Then, out of the $\left(\begin{array}{c}2 m \\ 3\end{array}\right)$ triangles defined by $P$, at least $(m+1) m(m-1) / 3$ contain $x$. (This minimum is achieved if $P \cup\{x\}$ is in general position, in particular if no two points of $P$ are collinear with $x$.)

Proof Let $p_{i} p_{j}$ be a directed segment joining two points of $P$, and let $d=(j-i)$ mod $2 m$. If $0 \leq d \leq m-1$, we call the segment $p_{i} p_{j}$ short; if $d=m$, we call it medium; and if $m+1 \leq d \leq 2 m-1$, we call it long.

A triangle $p_{i} p_{j} p_{k}$ with $i<j<k$ can have either three short sides, or two short sides and one medium side, or two short sides and one long side.

It is easy to see that all the triangles with three short sides contain $x$, and none of the triangles with one long side contain $x$. Furthermore, the triangles with one medium side can be grouped into pairs such that from each pair at least one triangle contains $x$ (exactly one triangle if $P \cup\{x\}$ is in general position). See Fig. 4 .

The number of triangles with three short sides is

$$
\frac{2 m}{3}(1+2+\cdots+(m-2))=\frac{m(m-1)(m-2)}{3},
$$

and the number of triangles with one medium side is $2 m(m-1)$. Thus, $P$ defines at least

$$
\frac{m(m-1)(m-2)}{3}+m(m-1)=\frac{(m+1) m(m-1)}{3}
$$




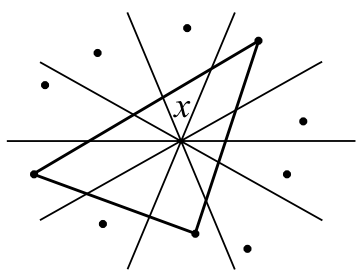

(a)

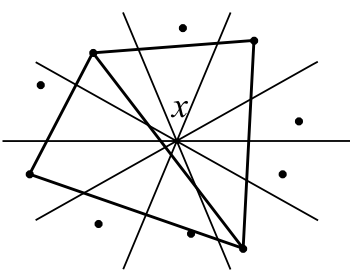

(b)

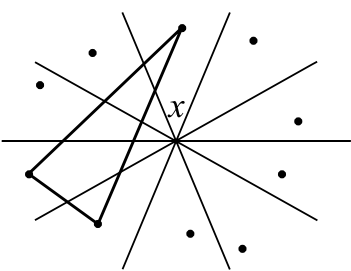

(c)

Fig. 4 (a) A triangle with three short sides always contains $x$. (b) The triangles with one medium side can be partitioned into pairs such that at least one triangle from each pair contains $x$. (c) A triangle with one long side never contains $x$

triangles that contain $x$ (exactly these many if $P \cup\{x\}$ is in general position).

Corollary 5.3 Let $h_{0}, \ldots, h_{m-1}$ be $m$ hyperplanes in $\mathbb{R}^{d}$ that pass through a common $(d-2)$-flat $\ell$ and divide space into $2 m$ parts. Let $P=\left\{p_{0}, \ldots, p_{2 m-1}\right\}$ be $2 m$ points, one from each part. Then $P$ defines at least $(m+1) m(m-1) / 3$ triangles that intersect $\ell$.

Proof of Theorem 1.6 Let $S$ be an $n$-point set in $\mathbb{R}^{d}$. By Corollary 5.1 there exist $2 d-1$ hyperplanes that pass through a common $(d-2)$-flat $\ell$ and partition $S$ into parts of size at most $n /(4 d-2)+O(1)$ each. We show that $\ell$ is our desired $(d-2)$ flat.

Each part has at least $n /(4 d-2)-O(1)$ points, so there are at least $\frac{n}{4 d-2}-$ $O(1))^{4 d-2}$ ways to choose $4 d-2$ points, one from each part.

By Corollary 5.3, each such choice of points defines at least $2 d(2 d-1)(2 d-2) / 3$ triangles that intersect $\ell$. On the other hand, each such triangle is counted at most $\left(\frac{n}{4 d-2}+O(1)\right)^{4 d-5}$ times. Thus the number of triangles intersected by $\ell$ is at least

$$
\begin{aligned}
& \left(\frac{n}{4 d-2}-O(1)\right)^{4 d-2} \cdot \frac{2 d(2 d-1)(2 d-2)}{3} /\left(\frac{n}{4 d-2}+O(1)\right)^{4 d-5} \\
& \quad=\frac{d^{2}-d}{6(2 d-1)^{2}} n^{3}-O\left(n^{2}\right)
\end{aligned}
$$

\section{Discussion}

The main open problem is to determine the exact value of the constants $c_{d}$ of the First Selection Lemma for $d \geq 3$. There remains a multiplicative gap of roughly $(d-1)$ ! between the current lower bound (1) and our upper bound (given by Theorem 1.3). We conjecture that Theorem 1.3 is tight and that the correct constants are $c_{d}=$ $(d+1)^{-(d+1)}$.

We suspect that the construction in Theorem 1.3 also witnesses sharpness of Theorem 1.6. But, to our embarrassment, we have been unable to find even the line that stabs most triangles in this construction for $d=3$. 


\subsection{A Generalization of Centerpoints}

Rado's Centerpoint Theorem [20] implies that for every $n$-point set $S$ in $\mathbb{R}^{d}$, there exists a $(d-2)$-flat $\ell$ that lies at depth $n / 3$ with respect to $S$, in the sense that every halfspace that contains $\ell$ contains at least $n / 3$ points of $S$. (Simply project $S$ into an arbitrary plane.)

But the $(d-2)$-flat $\ell$ of Corollary 5.1 lies at depth $(d-1) n /(2 d-1)-O(1)$ with respect to $S$. (Indeed, every halfspace that contains $S$ completely contains $2 d-2$ of the $4 d-2$ parts mentioned in Corollary 5.1.) We do not know whether this bound is tight.

This suggests the following generalization of Rado's Theorem: If $S$ is an $n$-point set in $\mathbb{R}^{d}$ and $0 \leq k<d$, then there always exists a $k$-flat at depth $\delta_{d, k} n$ with respect to $S$-a "center- $k$-flat"-for some constants $\delta_{d, k}$. As far as we know, the general question of determining these constants $\delta_{d, k}$ has not been explored. (The formula $\delta_{d, k}=(k+1) /(d+k+1)$ seems to fit all the currently known data. $)$

\subsection{From the First Selection Lemma to the Second}

The First Selection Lemma has been generalized by Bárány et al. [3], in conjunction with Alon et al. [1], and Živaljević and Vrećica [27] to the following result called the Second Selection Lemma in [16]:

If $S$ is an $n$-point set in $\mathbb{R}^{d}$ and $\mathcal{F}$ is a family of $m \leq\left(\begin{array}{c}n \\ d+1\end{array}\right) d$-simplices spanned by $S$, then there exists a point $p \in \mathbb{R}^{d}$ contained in at least

$$
c_{d}^{\prime}\left(\frac{m}{n^{d+1}}\right)^{s_{d}} n^{d+1}
$$

simplices of $\mathcal{F}$, for some constants $c_{d}^{\prime}$ and $s_{d}$ that depend only on $d$. (Note that $m / n^{d+1}=O(1)$, so the smaller the constant $s_{d}$, the stronger the bound.)

The Second Selection Lemma is an important ingredient in the derivation of nontrivial upper bounds for the number of $k$-sets in $\mathbb{R}^{d}$ (see [16, Chap. 11] for the definition and details). The derivation proceeds by "lifting" the lemma by one dimension, obtaining that if $\mathcal{F}$ is a family of $m d$-simplices spanned by $n$ points in $\mathbb{R}^{d+1}$, then there exists a line that stabs $\Omega\left(\left(\frac{m}{n^{d+1}}\right)^{s_{d}} n^{d+1}\right)$ simplices of $\mathcal{F}$.

Does this lifting step result in a loss of tightness? If we may make an analogy from the results of this paper, it seems that the answer is yes. (As we showed, $c_{2,0}=1 / 27$ by Theorem 1.3 , whereas $c_{3,1} \geq 1 / 25$ by Theorem 1.6.)

The current best bound for the Second Selection Lemma for $d=2$ is $\Omega\left(\mathrm{m}^{3} /\right.$ $\left(n^{6} \log ^{2} n\right)$ ), due to Eppstein $[11,19]$ (so $s_{2}$ can be taken arbitrarily close to 3 in (6)). On the other hand, we know that if $\mathcal{F}$ is a set of $m$ triangles in $\mathbb{R}^{3}$ spanned by $n$ points, there exists a line (specifically, a line determined by two points of $S$ ) that stabs $\Omega\left(m^{3} / n^{6}\right)$ triangles of $\mathcal{F}$ (see [10] and [21] for two different proofs of this fact). It might turn out that this logarithmic gap between the two cases is an artifact of the current proofs, but we believe that the three-dimensional problem does have a larger bound than the planar one.

Acknowledgement We would like to thank an anonymous referee for useful comments. 


\section{Appendix: The Planar Construction of Boros and Füredi}

Boros and Füredi [7] constructed a planar $n$-point set $\mathcal{P}_{n}$ for which, they claimed, no point $x \in \mathbb{R}^{2}$ is contained in more than $n^{3} / 27+O\left(n^{2}\right)$ triangles spanned by $\mathcal{P}_{n}$. There is a problem in their construction, however, and, as we show here, there exists a point $x$ contained in $\left(\frac{1}{27}+\frac{1}{729}\right) n^{3}$ triangles spanned by $\mathcal{P}_{n}$.

Their set $\mathcal{P}_{n}$ lies on the unit circle, and it consists of three clusters of $n / 3$ points, denoted $\mathcal{A}, \mathcal{B}$, and $\mathcal{C}$.

Each cluster is very small (sufficiently so), and the clusters are separated by an angular distance of roughly $2 \pi / 3$ from each other. The construction is not symmetric, however. The points in cluster $\mathcal{A}$ are uniformly separated, while the points in clusters $\mathcal{B}$ and $\mathcal{C}$ are separated by rapidly increasing distances (sufficiently rapidly so), with the distances increasing away from $\mathcal{A}$; see Fig. 5(a).

Now, let $x$ be a point in the convex hull of $\mathcal{B} \cup \mathcal{C}$. For each point $b \in \mathcal{B}$, trace a line $\ell_{b}$ from $b$ through $x$, until it intersects the unit circle again at $b^{\prime}$. Let $\mathcal{B}^{\prime}$ denote the set of these points $b^{\prime}$. If $\mathcal{B}$ is sufficiently small and $x$ is not too close to $\mathcal{B}$, then the lines $\ell_{b}$ will be almost parallel to each other.

The relative order between the points of $\mathcal{B}^{\prime}$ and the points of $\mathcal{C}$ along the unit circle determines the number of triangles of the form $\mathcal{A B C}, \mathcal{B B C}$, and $\mathcal{B C C}$ that contain $x$. (By a triangle of the form $\mathcal{A B C}$ we mean a triangle $a b c$ with $a \in \mathcal{A}, b \in \mathcal{B}, c \in \mathcal{C}$; and so on.)

In fact, each triangle of the form $\mathcal{A B C}$ containing $x$ corresponds to a triple $a b^{\prime} c$, with $a \in \mathcal{A}, b^{\prime} \in \mathcal{B}^{\prime}, c \in \mathcal{C}$ such that $c$ is farther from $a$ than $b^{\prime}$. Similarly, each triangle of the form $\mathcal{B B C}$ containing $x$ corresponds to a triple $b_{1}^{\prime} b_{2}^{\prime} c$ with $b_{1}^{\prime}, b_{2}^{\prime} \in \mathcal{B}^{\prime}$, $c \in \mathcal{C}$, where $c$ lies between $b_{1}^{\prime}$ and $b_{2}^{\prime}$; and each triangle of the form $\mathcal{B C C}$ containing $x$ corresponds to a triple $b^{\prime} c_{1} c_{2}$ with $b^{\prime} \in \mathcal{B}^{\prime}, c_{1}, c_{2} \in \mathcal{C}$, where $b^{\prime}$ lies between $c_{1}$ and $c_{2}$.

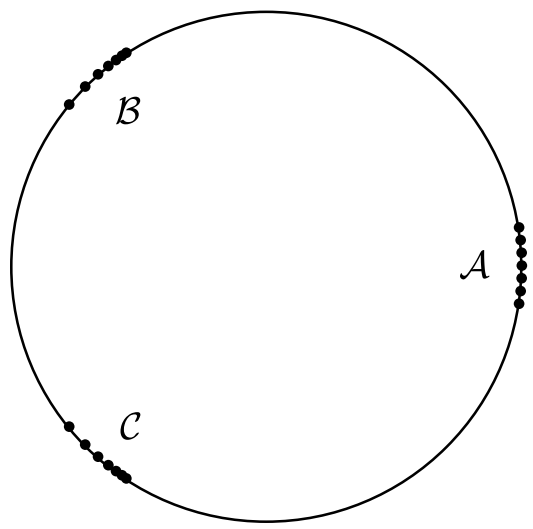

(a)

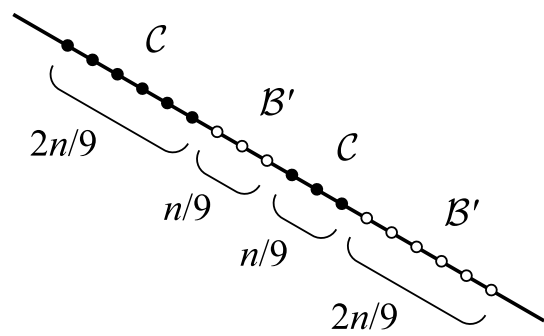

(b)

Fig. 5 (a) The set $\mathcal{P}_{n}$ of Boros and Füredi. (b) Relative order of the points of $\mathcal{B}^{\prime}$ (white circles) and $\mathcal{C}$ (black circles) along the unit circle (which appears here almost as a straight line) 
Note that the distances between the points of $\mathcal{B}^{\prime}$ increase rapidly towards $\mathcal{A}$. Also note that moving the point $x$ towards or away from $\mathcal{B}$ has the effect of enlarging or shrinking the image $\mathcal{B}^{\prime}$, while moving $x$ sideways has the effect of moving $\mathcal{B}^{\prime}$ sideways.

Therefore, it is not hard to see, we can position the point $x$ such that the order of the points in $\mathcal{B}^{\prime} \cup \mathcal{C}$ reading towards $\mathcal{A}$ is: $2 n / 9$ points of $\mathcal{C}$, followed by $n / 9$ points of $\mathcal{B}^{\prime}$, followed by $n / 9$ points of $\mathcal{C}$, followed by $2 n / 9$ points of $\mathcal{B}^{\prime}$; see Fig. 5 (b).

It follows that $x$ is contained in $\frac{8}{243} n^{3}$ triangles of the form $\mathcal{A B C}, \frac{2}{729} n^{3}$ triangles of the form $\mathcal{B B C}$, and $\frac{2}{729} n^{3}$ triangles of the form $\mathcal{B C C}$. Thus, $x$ is contained in atal of $\frac{28}{729} n^{3}=\left(\frac{1}{27}+\frac{1}{729}\right) n^{3}$ triangles.

On the other hand, it can be checked that this point $x$ is the one that stabs asymptotically the maximum number of triangles. Hence, this construction gives a bound of $c_{2} \leq 1 / 27+1 / 729$.

\section{References}

1. Alon, N., Bárány, I., Füredi, Z., Kleitman, D.J.: Point selections and weak $\epsilon$-nets for convex hulls. Comb. Probab. Comput. 1, 189-200 (1992). http://www.math.tau.ac.il/ nogaa/PDFS/abfk3.pdf

2. Bárány, I.: A generalization of Carathéodory's theorem. Discrete Math. 40, 141-152 (1982)

3. Bárány, I., Füredi, Z., Lovász, L.: On the number of halving planes. Combinatorica 10(2), 175-183 (1990). http://www.cs.elte.hu/ lovasz/morepapers/halvingplane.pdf

4. Basu, S., Pollack, R., Roy, M.-F.: Algorithms in Real Algebraic Geometry, 2nd edn. Algorithms and Computation in Mathematics, vol. 10. Springer, Berlin (2006)

5. Buck, R.C., Buck, E.F.: Equipartition of convex sets. Math. Mag. 22, 195-198 (1948-1949)

6. Boros, E., Füredi, Z.: Su un teorema di Kárteszi nella geometria combinatoria. Archimede 2, 71-76 (1977)

7. Boros, E., Füredi, Z.: The number of triangles covering the center of an $n$-set. Geom. Dedic. 17, 69-77 (1984)

8. Bukh, B.: A point in many triangles. Electron. J. Combin. 13(1), Note 10, 3 pp. (2006), (electronic)

9. Ceder, J.G.: Generalized sixpartite problems. Bol. Soc. Mat. Mex. (2) 9, 28-32 (1964)

10. Dey, T.K., Edelsbrunner, H.: Counting triangle crossings and halving planes. Discrete Comput. Geom. 12(1), 281-289 (1994)

11. Eppstein, D.: Improved bounds for intersecting triangles and halving planes. J. Comb. Theory Ser. A 62(1), 176-182 (1993). http://www.ics.uci.edu/ eppstein/pubs/Epp-TR-91-60.pdf

12. Fadell, E.R.: Ideal-valued generalizations of Ljusternik-Schnierelmann category, with applications. In: Fadell, E., et al. (eds.) Topics in Equivariant Topology. Sém. Math. Sup., vol. 108, pp. 11-54. Press Univ. Montréal, Montréal (1989)

13. Fadell, E.R., Husseini, S.: An ideal-valued cohomological index theory with applications to BorsukUlam and Bourgin-Yang theorems. Ergod. Theory Dynam. Sys. 8, 73-85 (1988)

14. Inoue, A.: Borsuk-Ulam type theorems on Stiefel manifolds. Osaka J. Math. 43(1), 183-191 (2006). http://projecteuclid.org/euclid.ojm/1146243001

15. Kárteszi, F.: Extremalaufgaben über endliche Punktsysteme. Publ. Math. (Debr.) 4, 16-27 (1955)

16. Matoušek, J.: Lectures on Discrete Geometry. Springer, New York (2002)

17. Matoušek, J.: Using the Borsuk-Ulam Theorem. Springer, Berlin (2008). Revised 2nd printing

18. Moon, J.W.: Topics on Tournaments. Holt, Rinehart and Winston, New York (1968)

19. Nivasch, G., Sharir, M.: Eppstein's bound on intersecting triangles revisited. J. Comb. Theory Ser. A 116(2), 494-497 (2009). arXiv:0804.4415

20. Rado, R.: A theorem on general measure. J. Lond. Math. Soc. 21, 291-300 (1947)

21. Smorodinsky, S.: Combinatorial problems in computational geometry. Ph.D. thesis, Tel Aviv University, June 2003. http://www.cs.bgu.ac.il/ shakhar/my_papers/phd.ps.gz

22. Wagner, U.: On $k$-sets and applications. Ph.D. thesis, ETH Zürich, June 2003. http://www.inf.ethz. $\mathrm{ch} / \sim$ emo/DoctThesisFiles/wagner03.pdf 
23. Welzl, E.: Entering and leaving $j$-facets. Discrete Comput. Geom. 25, 351-364 (2001). http://www. inf.ethz.ch/personal/emo/PublFiles/EnterLeave_DCG25_01.ps

24. Wendel, J.G.: A problem in geometric probability. Math. Scand. 11, 109-111 (1962)

25. Živaljević, R.T.: User's guide to equivariant methods in combinatorics II. Publ. Inst. Math. (Beogr.) 64(78), 107-132 (1998)

26. Živaljević, R.T.: Topological methods. In: Goodman, J.E., O’Rourke, J. (eds.) Handbook of Discrete and Computational Geometry, 2nd edn. CRC Press, Boca Raton (2004). Chap. 14

27. Živaljević, R.T., Vrećica, S.T.: The colored Tverberg's problem and complexes of injective functions. J. Comb. Theory Ser. A 61(2), 309-318 (1992) 